\title{
Exploration of Relation between Accomplice Withdrawal and Discontinuance and Their Application in China's Criminal Law
}

\author{
Shuang Zhang \\ Hainan Vocational College of Political Science and Law, Haikou Hainan, 571000, China
}

Keywords: Accomplice withdrawal, Accomplice discontinuance, Relation, Criminal law, Application.

\begin{abstract}
The original intention of accomplice withdrawal is to make up the existing deficiencies in accomplice discontinuance system and solve the problem that doer carries out withdrawal but can not stop accomplished offense and results in criminal liability. However, during the further study of accomplice withdrawal theory, it gradually becomes an independent theory that differs from accomplice discontinuance and fully shows its advantages. However, in our current criminal law, there are still no relevant articles of law about accomplice withdrawal. Thus, in juridical practice, there is no specific legal ground to the punishment of accomplice withdrawal. As a result, accomplice withdrawal theory should be introduced to our criminal law, realizing sinicization.
\end{abstract}

\section{Relation Analysis between Accomplice Withdrawal and Discontinuance}

\section{Relation between Accomplice Withdrawal and Discontinuance}

Similar important conditions of timeliness

In the legal contents of our country, it is not explicitly stipulated accomplice discontinuance in important conditions of timeliness, and in practice, it only refers to the independent stipulation. In short, it means that there is tenable condition of discontinuance at random time horizon before accomplishment of a crime. However, once after accomplishment of a crime, such condition is gone $^{[1]}$. But the accomplice withdrawal needs to set the timeliness aspect before accomplishment, the period of preparation and implementation for a crime.

Similar subjectivity

In accomplice discontinuance, requirement to the doer is to discontinue crimes with his own willing, which is also the randomicity requirement. That is to say, what can be regarded as discontinuance is that the doer is able to finish crime individually but gives up to do so with his own willing. However, the requirement to accomplice withdrawal is rather random, which is same with discontinuance. As for the randomicity affirmation, from the subjective angle, even though the crime can not be finished, as long as the doer thinking that can be done but giving up could reflect the corresponding randomicity. On the contrary, from the objective angle, if the doer could finish the crime but he thinks the can not and gives up, such condition doesn't have randomicity.

Similar objective behavior

As to the behavioral expression, accomplice withdrawal and discontinuance are the same. And the requirement of accomplice discontinuance to the doer refers to the discontinuance behavior to prevent other accomplices keeping criminal behavior or prevent the criminal result. Under this circumstance, other accomplices don't keep criminal behavior or criminal result doesn't happen. However, accomplice withdrawal asks seceder to escape from the existing accomplice relation, and meanwhile influence of seceder to the implementing behavior doesn't happen. Only if the above requirement is reached can the doer be regarded as escaping from the accomplice relation ${ }^{[2]}$. According to the above analysis, such two behaviors as accomplice discontinuance and withdrawal need the doer to prevent other accomplices keeping criminal behavior or prevent the criminal result. Even thought there are differences between these two behavioral outcomes, it has same objective expression. 


\section{Differences between Accomplice Withdrawal and Discontinuance}

\section{Effectiveness requirement}

In the accomplice discontinuance, it needs some effectiveness and the doer must prevent other accomplices' criminal behavior or stop the criminal result, which is exactly the biggest difference from discontinuance and accomplishment. In addition, this is also the most obvious watershed between accomplice withdrawal and discontinuance. In the accomplice discontinuance, the specific requirement is that there are no accomplished results. However, accomplice withdrawal has essential difference from it, with accomplished results. In general, on the basis of randomicity and effectiveness, it could only realize accomplice discontinuance. However, as long as it lacks effectiveness, the discontinuance would not come into existence. However, this is exactly the important space for accomplice withdrawal system to exist and develop. But this also makes most scholars to consider accomplice withdrawal as a relief measure in accomplice discontinuance system. Number of people that can quit

Accomplice discontinuance includes two patterns as the discontinuance caused by several doers or all doers. That is to say, there are two reasons avoiding the harmful consequences: firstly, some doers carry out discontinuance behaviors and prevent other accomplices' criminal behavior, finally avoiding criminal results; secondly, all doers give up the managed criminal behaviors at the same time and finally prevent the occurrence of criminal results. In the above two conditions, as long as there is discontinuance behavior, the accomplice could be regarded as discontinuance ${ }^{[3]}$. However, premise of accomplice withdrawal is that both harmful consequence and accomplishment of a crime have happened. Thus, there is no such condition that all accomplices withdraw from accomplice relation at the same time in accomplice withdrawal. In short, only when part doers carry out withdrawal behaviors and harmful consequence happens can it possesses withdrawal condition.

Location of theory of crime

According to the above elaboration contents, it finds that accomplice withdrawal belongs to the independent problem in the theory of accomplice. However, the essence of accomplice discontinuance is always the stop condition of criminal, which should be mentioned in the same breath with preparation, accomplishment and not accomplishment for a crime. It should be put into discontinuance rather than the theory of accomplice.

Legal nature

From the aspect of law, annulling a punishment of accomplice discontinuance should be decided according to the social extent of injury of discontinuance doers. And the detailed behavior to lower social harmfulness is the reduction of objective harms and subjective malignance reduction of doers to the society or victims' legal interest. This belongs to the concept of unified subjectivity and objectives. However, as for the essence, it is due to the cutting off the relation between behavior and result by discontinuance doers, finally preventing the occurrence of result. Reviewing the accomplice withdrawal, it also possesses reduction of subjective culpability of the mind and gradual change to objective behavior. However, it hasn't prevented accomplishment of a crime. Thus, there is no such purpose to reduce harms to society or victims' legal interest. Certainly, according to the above analysis and study, it could find that accomplice withdrawal needs tolerance in law and the main reason is that in the withdrawal occasion, even the doer doesn't totally cancel the legal interest harms or cut off the relation between behavior and result, its practical illegal responsibility has been lowed. Thus, this is a just evaluation to the withdrawal doers because he has tried best to prevent others. This shows that accomplice withdrawal has the objective expression of discontinuance. However, there is difference to discontinuance in legal nature. Thus, the punishment to withdrawal would usually be more serious than that to discontinuance ${ }^{[4]}$. 


\section{Study of Application to Accomplice Withdrawal in China's Criminal Law}

\section{Affirmation and Analysis of Accomplice Withdrawal before Starting}

\section{Instigator's withdrawal}

Instigation mainly refers to the behavior to induce others form criminal intent. And the withdrawal problem of accomplice relation of instigator before starting is the key problem with rather big controversy among those experts, with detailed manifestation in the difference of nature affirmation. That is to say, there is some difference between accomplice independence and dependence. Thus, it also includes independent and dependent instigation. However, in China's criminal law, there is punishable natures in preproduction phase and as long as doer accepts instigation, the instigator has have the condition of accomplice withdrawal after the preproduction phase.

From the narration to accomplice relation, the instigator needs to express individual willing to give up criminal and meanwhile to make doer give up criminal, such as persuasion, etc. However, only such persuasion behavior is not enough, which can not be regarded as withdrawal. Main reason is that the doer doesn't listen to the instigator and keeps carrying our criminal. Such withdrawal could lead to harms to others. Thus, on the basis of the above behaviors, instigator should adopt further preventing behavior, such as going to the police, physically prevention, etc. If the doer gives up the implementation of instigation behavior but carry out the original behavior, as to such accomplice relation, the danger caused by instigator can be regarded as disappearing as well as the original accomplice relation. And the following behavior of the doer is carried out by new criminal intention. Thus, instigator can be regarded as withdrawal.

Helper's withdrawal

Help refers to the doer with criminal intention, whose criminal behavior can be easily done. And the helper is the people helping others' crime ${ }^{[5]}$. In our criminal law, helper belongs to accessory offender. There are two helping ways: firstly, to provide material help to the doers; secondly, to provide mental stimulation to doers. In the first way, if the helper wants to make withdrawal, he needs to withdraw the provided material support. If it is lethal weapon, the helper should take back to avoid the occurrence of accomplice relation and finally cancel the relation. The second way is to provide mental help. If want withdrawal, the helper should cancel the stimulation and intensification behaviors. However, helper plans to offer the doer help and during the doing process, he helps to look out or escape. Then it only needs to cancel the oral agreement and ensure the doer realize that. If it is only stimulation, it just needs persuasion and discontinuance. But whether the doer is willing to give up criminal intention or no is hard to ensure.

Joint doers withdrawal

During the process of studying the withdrawal problem to joint doers, the most important is to ensure the existence of those joint doers, collusive coprincipals in theory of criminal law. Collusive coprincipals refer to more than two people to implement crimes, and some of them are willing to withdrawal before starting while others don't. Under such circumstances, it should be dealt with by their position in accomplice and according to the instigator or helper withdrawal. However, from the angle of joint doers, only the crimes with more than two doers belong to joint doe. Thus, there are no joint doers before starting and it finally needs to revert to helper or instigator. Even though collusive coprincipals are acknowledged, they are the same in essence. That is to say, discontinuance before starting still refers to helper or instigator, which means withdrawal before starting being same with helper or instigator. Thus, there are two distinguishes to affirm accomplice withdrawal: firstly, under the circumstance of instigation condition, acknowledgement withdrawal is same with instigator withdrawal, which should possess not only withdrawal intents and behaviors but also canceling others' criminal intents. Secondly, under the circumstance of helping form joint offender, it needs to cancel the influence to the result. At the same time, during the process of starting, he should be just joining in accomplice. 


\section{Affirmation and Analysis of Accomplice Withdrawal after Starting}

Under the circumstance of starting crimes, as long as it happens, the possibility of damaging result is very strong. At the same time, the implementation behavior has damaged others' legal interest. And during the implementation, accomplices are mutual to each other, with stable cooperative relation. Thus, after starting, it is hard to affirm accomplice withdrawal. But the possibility still exists, because some doers may propose withdrawal intents and stop others' behavior, finally avoiding criminal results. Under such circumstance, it can affirm the accomplice withdrawal of doers who don't need to take corresponding criminal responsibility for the others' following criminal behavior or the finally criminal results. It needs to keep intensifying the withdrawal after starting to further promote deterrence and provide fallback position for those penitential doers, lowering the extent of injury caused by criminal behaviors.

\section{Conclusion}

To sum up, the accomplice relation withdrawal refers to the behavior that some doers cut off and withdraw relation to other accomplices but others keep implementing crimes and cause criminal results, on the basis of current accomplice relation and before accomplishment of a crime. There are many similarities as well as differences between accomplice withdrawal and it. Thus, this article analyzes those similarities and differences, studies the suitable part of accomplice withdrawal in our active criminal law and finally ensure it rationally applied to our country by further studying the theory of accomplice withdrawal.

\section{References}

[1] Yao Wanqin, Chen Liyi, On Withdrawal of Accomplice Relation - with Accomplice Discontinuance as Perspective, Zhengfa Xuekan, 2013, 30(1):87-93.

[2] Niu Fanqi, Wang Xiaoyu, Comparative Study to Accomplice Withdrawal and Enlightenment to Our Juridical Practice, Knowledge Economy, 2012(14):27-27.

[3] Zhao Qianqian, On the Affirmation and Judicial Application of Accomplice Withdrawal, Theory Research, 2013(17):134-135.

[4] Liu Yanhong, Benchmark of Judgment of Accomplice Withdrawal: Normative Causal Relationship Interruption Theory, Peking University Law Journal, 2013, 25(4):747-765.

[5] Huang Zeyu, Affirmation and Establishment Requirement f Accomplice Withdrawal, Today Hubei (late period), 2014(1):19-19. 